\title{
Computational study on lateral and medial wear characterization in knee implants by a multibody dynamic system
}

Received: 16 April 2020 / Revised: 25 September 2020 / Accepted: 23 October 2020 / Published online: 8 January 2021

(C) The Author(s) 2020

\begin{abstract}
Wear is one of the main mechanical factors that limits the survival of total knee replacements (TKRs) and it is known to be highly dependent on the local kinematics of the knee joint. In this study, an analytical wear model was coupled to a multibody dynamic model to obtain wear distribution at the lateral and medial contact plateaus of different TKRs. The major aim was to analyze if wear distribution on the contact plateaus can be an indicator of elevated tibiofemoral misalignment which can lead to rapid TKR failure. For the multibody dynamic simulations, commercial and prototype TKR geometries were used, coupled with an augmented Archard's law. Squat movement was chosen due to its importance both in sports and in everyday life. As a conclusion, a new parameter, denoted as wear imbalance, is introduced, which can indicate whether a TKR, due to its geometrical features, is prone to be subjected to elevated wear and failure.
\end{abstract}

\section{Introduction}

Due to the constant development of total knee replacements (TKRs), their reliability continuously improves, but failures still occur. According to the relevant literature, major causes of failure can be credited to joint infection, loose components and wear between TKR components [1]. Regarding wear-related challenges, numerous studies, both theoretical and experimental works, have been seeking those certain mechanical phenomena or material properties, which exhibit key importance on the understanding of re-occurring elevated wear propagation. It is worth noting that these wear-inducing phenomena or parameters can be divided into direct and indirect ones.

The most influencing and researched direct wear-inducing parameters are the load and the sliding-velocity (or path), however, serious emphasis is paid to the slide-roll ratio as well. This ratio is often considered constant, based on the study of McGloughlin and Kavanagh [2], and it has been frequently considered and applied on pin-on-disc test rigs or knee simulators [3-5]. It must be added that the application of constant slide-roll ratio has only been proven correct if it was applied on simple pin-on-disc or ball-on-disc tests, where the kinematics could be precisely adjusted to a specific constrained configuration. For this reason, several papers show that the idea of constant slide-roll ratio might not be precise enough in case of TKRs $[6,7]$ due to their complex geometry and unconstrained motion, which highly alter the local kinematics [8].

Among the indirect parameters [9], it is worth mentioning the insert thickness [10], the rotational alignment of the tibial component [11], the tibiofemoral alignment (TFA) or the lack of this alignment [12]. It is known

G. Fekete $(\varangle)$

Savaria Institute of Technology, Faculty of Informatics, Eötvös Loránd University, Károlyi Gáspár tér 4, Szombathely 9700, Hungary

E-mail: fg@inf.elte.hu

G. Fekete

Research Academy of Grand Health, Faculty of Physical Education, Ningbo University, Fenghua Road 818, Ningbo 315211, China 
that if these parameters fall short to remain in a certain range, they become wear-inducing factors. However, it is unidentified how they are interlinked with wear propagation or wear distribution.

In order to answer the above-mentioned pending questions, a few papers have been published where the authors investigated, with analytical $[13,14]$ or finite element methods $[10,15]$, how TKR wear is affected by non-constant slide-roll ratio and non-constant load, based on an augmented version of Archard's law [16].

In spite of the original form of Archard's law being based on a simple linear equation, it is still widely used in the contemporary literature $[17,18]$, since the predicted wear is in reasonable agreement with regard to the trend and magnitude of other more complicated models [19,20].

It should be also noted that the aforementioned analytical models $[13,14]$ only considered wear in a single-point contact, which subsequently required a more extensive numerical study on wear distribution. Among several methods, the multibody dynamic (MBD) approach is seen as an adequate direction. Flores [21] demonstrated in his model how the wear phenomenon, in case of revolute clearance joints, could be modeled as a combination of a multibody dynamic system coupled with Archard's law of wear propagation. Based on Flores approach, a combination of a multibody dynamic model and a new wear extension tool can address wear propagation in multiple points on both sides of the tibia plateau.

Therefore, in this paper, the first aim was to provide such a hybrid numerical-analytical tool, which not only provides an augmented wear model, but also enables us to locally differentiate wear propagation and distribution between the lateral and medial compartments of any of TKR. As a second aim, a new parameter is introduced which can answer the question whether uneven wear distributions on the contact plateaus could lead to elevated tibiofemoral misalignment, accompanied with rapid TKR failure.

As a motion of interest, squat movement was chosen for this study due to two reasons. First, during squatting, the two most influencing wear parameters [13] reach their maximum values: the contact forces [22] and the slide-roll ratio [7]. Second, squat is a widely applied exercise for rehabilitation and for thigh muscles strengthening in view of sport or medical reasons [23-25]. Therefore, it is essential to know how TKRs are affected by wear if this motion is carried out in the long term.

\section{Methods}

The algorithm, which allows us to estimate and draw conclusions about wear propagation on the lateral and medial side of any TKR, consists of five separated steps (Fig. 1).

First, the governing equations are introduced. In general, an ordinary differential equation (ODE), with regard to the wear model, and a differential algebraic equation (DAE) system, with regard to the MBD model, must be solved separately. This is followed by the description of the applied wear model. Here, the wearinfluencing parameters are listed and applied. Thereafter, multibody dynamic simulations are carried out to obtain the lateral and medial contact forces. In this part, the fundamental features of TKRs are discussed together with the applied boundary conditions and mechanical constraints. Subsequently, wear imbalance is introduced as a new parameter. Finally, the contact forces, obtained from the MBD simulations, are incorporated into the wear model to yield the results, which is explained in detail in the following.

\begin{tabular}{|l|l|}
\hline 1. & - Introduction of the governing equations: the concept \\
\hline 2. & - Description of the applied wear model \\
\hline 3. & - Multibody dynamic simulations \\
\hline 4. & - Introduction of wear imbalance \\
\hline 5. & - Results: Integration of the MBD results into the analytical wear model \\
\hline
\end{tabular}

Fig. 1 Flowchart about modeling steps 
Table 1 Wear parameters

\begin{tabular}{ll}
\hline Parameter & Quantity/function \\
\hline$k:$ specific wear rate [3] & $1.3 \times 10^{-6}\left[\mathrm{~mm}^{3} / \mathrm{Nm}\right]$ \\
$\mu_{k}:$ average coefficient of kinetic friction [26] & $0.0575[-]$ \\
BW: Body weight & $800 \mathrm{~N}$ \\
$F_{t f}(t):$ tibiofemoral force relative to $B W[22]$ & $0.7702 \cdot t^{3}+0.554 \cdot t^{2}+1.6244 \cdot t+1.0311[-]$ \\
$S / R(t):$ Slide-roll ratio [7] & $-0.111667 \cdot t^{3}+0.3631732 \cdot t^{2}+0.02378365 \cdot t+1.137558[-]$ \\
$v_{C T t}:$ tangential velocity of the tibia in the contact & $30[\mathrm{~mm} / \mathrm{s}]$ \\
plateau [26] & \\
$d t:$ duration of motion & Defined by the motion [s] \\
\hline
\end{tabular}

\subsection{Introduction of the governing equations: the concept}

In case of any MBD system, a DAE must be solved, which is generally in the form

$$
\left[\begin{array}{cc}
\mathbf{M} & -\mathbf{D}^{\mathbf{T}} \\
\mathbf{D} & \mathbf{0}
\end{array}\right] \cdot\left[\begin{array}{c}
\ddot{\mathbf{x}} \\
\lambda
\end{array}\right]=\left[\begin{array}{l}
\mathbf{f} \\
\gamma
\end{array}\right]
$$

where $\mathbf{M}$ is the mass matrix, $\mathbf{D}\left(-\mathbf{D}^{\mathbf{T}}\right)$ is the Jacobian matrix (and its transpose) of the constraint equations, $\ddot{\mathbf{x}}$ includes the acceleration terms, $\lambda$ is the Lagrange multiplier (representing the constraint-, and contact forces), while $\mathbf{f}$ and $\boldsymbol{\gamma}$ are the external forces/moments and inertial terms, respectively.

The MSC.ADAMS software can simultaneously solve Eq. (1) to $\ddot{\mathbf{x}}$ and $\lambda$ if the modeling steps (definition of geometry-, material-, constraints and loads) are carried out. For wear modeling, the term $\lambda$ is needed, which includes the contact forces $\left(\mathbf{F}_{\mathbf{C}}(\mathrm{t})\right)$ acting on the tibia plateaus. If these contact forces are determined, then a simple ODE (Eq. (2)) must be solved to calculate wear:

$$
d W=f(F(t) s(t) k, \ldots),
$$

where $F(t)$ represents a general load function. This function is to be exchanged by the contact force function $\left(\mathbf{F}_{\mathbf{C}}(\mathrm{t})\right)$ obtained from $\lambda$, to yield wear propagation on both sides of the tibia plateau.

\subsection{Description of the applied wear model}

In the form of an ODE, a new analytical wear model has been deduced [13], which can estimate the propagation of wear volume by incorporating both the load and the slide-roll ratio as a function of time:

$$
d W=k \cdot \mu_{k} \cdot \mathrm{BW} \cdot F_{t f}(t) \cdot v_{C T t}(t) \cdot S / R(t) \cdot d t .
$$

The parameters of Eq. (3) are listed in Table 1.

\section{Multibody dynamic simulations}

To obtain the contact forces, three TKRs have been examined with regard to their wear propagation (Fig. 2). Two of them were middle-sized TKRs produced by BioTech: one posterior stabilized (PS), and one cruciate retained type (CR). The third TKR is a large-sized prototype prosthesis [27] (Fig. 2). It must be noted that the prototype TKR has an asymmetric insert, while the PS and CR are equipped with symmetric ones. Although post-cam design has not been investigated, it can be declared that the method is unaffected by the different contact surfaces (flat-to-flat or curve-to-curve); therefore, it is generally applicable. The applicability was demonstrated on the types of inserts as well, since in this study both symmetric and asymmetric trays were applied and investigated.

It should be pointed out that flexible elements can also be analyzed in MSC.ADAMS to consider elastic or plastic deformation; however, this topic was not examined in this paper.

As the first step of modeling, the TKR geometries have been 3D scanned, and the geometrical models were imported into the MSC.ADAMS multibody dynamic system (Fig. 3). 


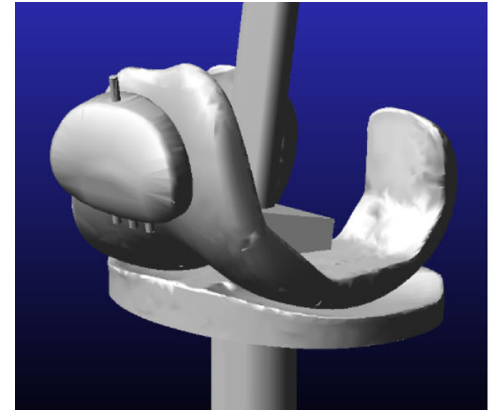

Cruciate retaining $(\mathrm{CR})$

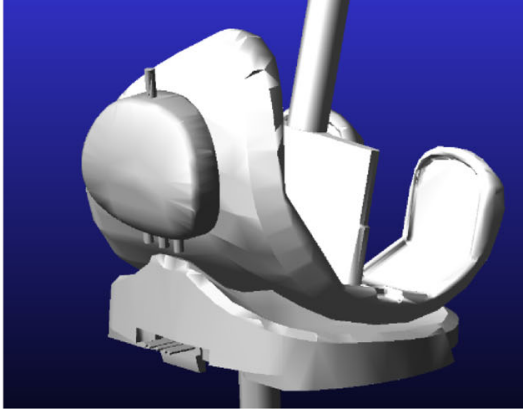

Posterior stabilized (PS)

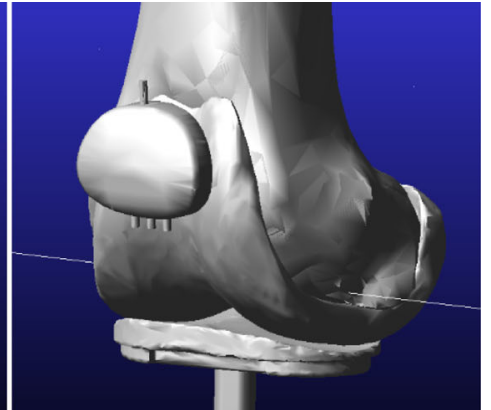

Prototype

Fig. 2 The different TKR geometries

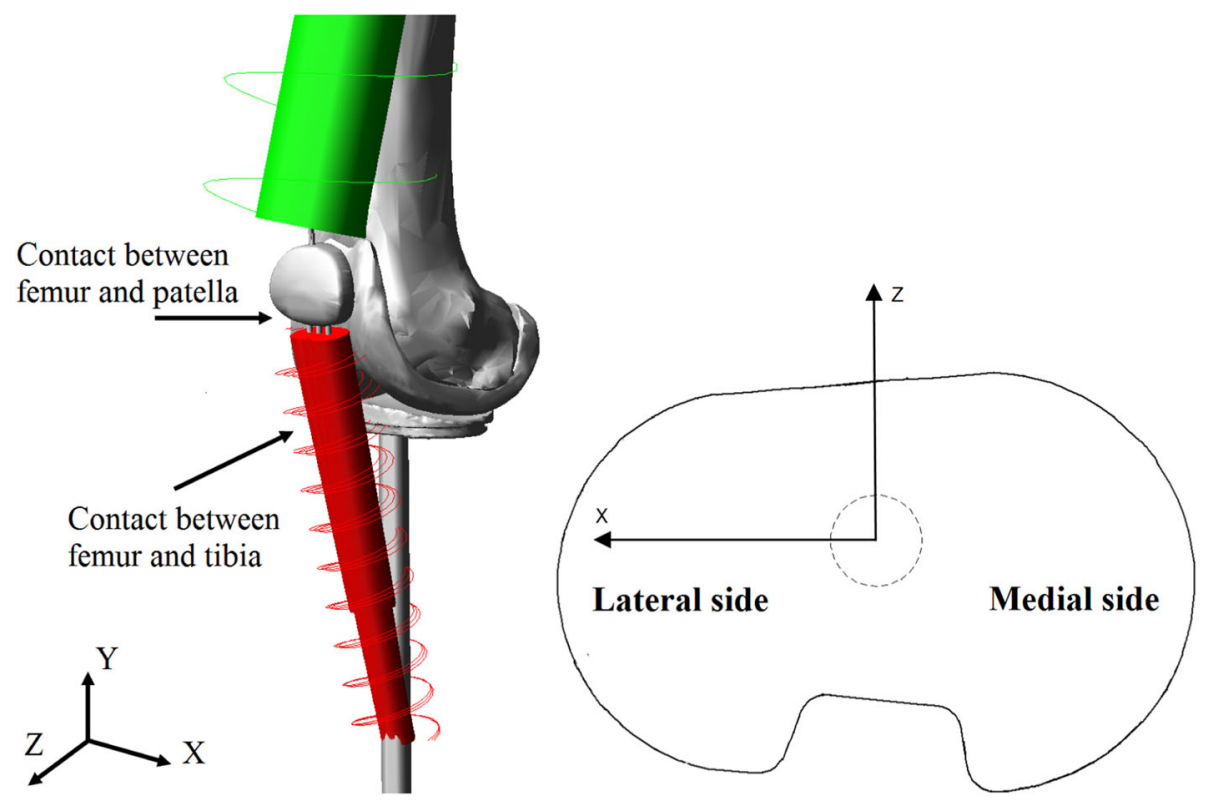

Fig. 3 The applied MBD model

Table 2 The applied boundary conditions on the MBD models

Hard tissues

Soft tissues

Kinematic constraints

Kinetic constraints

Contact constrains
Tibia, patella and femur were assumed as rigid bodies with regard to all MBD models (CR, PS, Prototype)

The patellar ligament and the quadriceps muscle were modeled with TRANSLATIONAL SPRING-DAMPER (Fig. 3). The stiffness coefficient was set to $130 \mathrm{~N} / \mathrm{mm}$, while the damping coefficient to $0.15 \mathrm{Ns} / \mathrm{mm}$ for both springs according to the measured values in the literature $[28,29]$

The femur distalis was constrained to perform only translational motion along the $y$ axis by a GENERAL POINT MOTION constraint. The ankle part of the model was constrained by a SPHERICAL JOINT, allowing rotation about all axes, but no translations. By applying this constraint, the tibia can carry out a natural, nonrestrained rotation

A FORCE VECTOR was applied on the femur distalis representing the load of the body weight (BW). This vector remained constantly orthogonal to the global $x$ axis. Its magnitude was set to $800 \mathrm{~N}$, which represents the average male weight in Hungary or in North America [30,31]. It must be mentioned that comparing forces to BW or using $1 \mathrm{BW}$ as load is an internationally accepted approach [32,33]

Between the femur, tibia and patella, CONTACT constraints were set according to Coulomb's law with respect to the kinetic friction coefficient $\left(\mu_{k}=0.0575\right)$ which was obtained from experiments [26]. It must be mentioned that the contact force is not orthogonal to the surface, since it has a friction component as well 


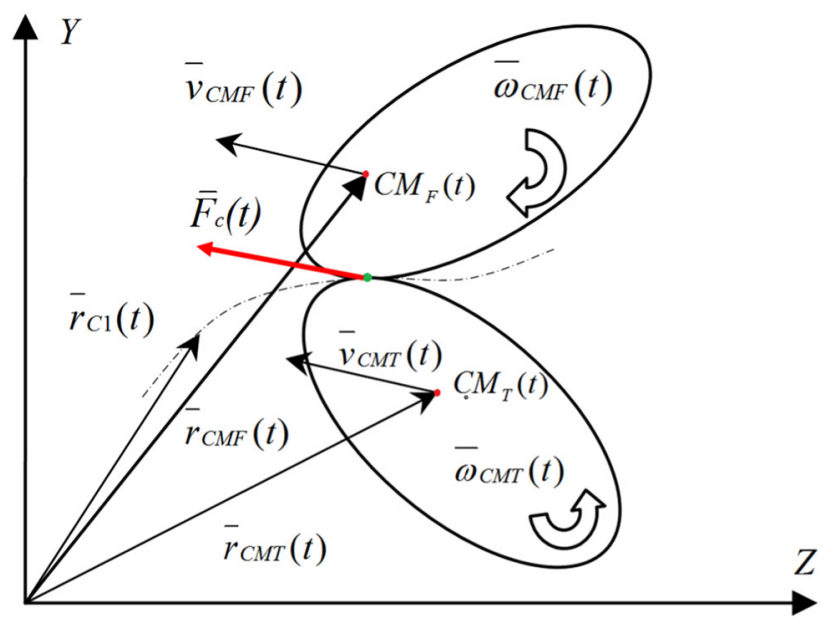

Fig. 4 Kinematics and kinetics of the contacting bodies

Boundary conditions were applied to each model identically (Table 2). The below-mentioned constraints are indicated the same way as they are referenced in the MSC.ADAMS software.

In the following, the necessary kinematical and kinetic quantities are listed, which can be directly obtained from the simulations as functions of time:

- $\mathbf{r}_{\mathbf{C 1}}(t)$ position vector: It describes the instantaneous position of the contact points with regard to the two bodies, defined in the absolute coordinate system. If $i=1$, contact between femur and tibia is considered (Fig. 4).

- $\mathbf{F}_{\mathbf{c}}(t)$ contact force vector. It describes the normal $\left(F_{c n}(t)\right)$ and friction $\left(F_{c s}(t)\right)$ components of the forces in the instantaneous contact positions (Fig. 4).

During the simulation, contact is made between the lateral and medial part of the tibia plateau and the femoral surface. The MSC.ADAMS simultaneously detects and stores the contacts $\left(\mathbf{r}_{\mathbf{C 1}}(t)\right)$ and contact forces $\left(\mathbf{F}_{\mathbf{c}}(t)\right)$ for later evaluation. The obtained position vectors allow us to differentiate the lateral and medial sides and the location of the contact forces $\left(F_{c n-\text { lateral }}\right.$ and $\left.F_{c n-m e d i a l}\right)$ on the plateau. These force functions, as functions of time, serve as inputs for the augmented Archard's law to calculate wear volume on the lateral and medial sides of the tibia plateau:

$$
d W_{\text {lateral }}=k \cdot \mu_{k} \cdot F_{\text {cn-lateral }}(t) \cdot v_{C T t}(t) \cdot S / R(t) \cdot d t
$$

and

$$
d W_{\text {medial }}=k \cdot \mu_{k} \cdot F_{\mathrm{cn}-\text { medial }}(t) \cdot v_{C T t}(t) \cdot S / R(t) \cdot d t .
$$

It must be mentioned that the numerical force function $F_{c n}(t)$ is not a relative force, unlike in the original equation (3); therefore, the differential equation is not multiplied by the bodyweight. During the simulations, $d t$ has been set to $1.5 \mathrm{~s}$, which is the approximate duration of a squat cycle (flexion-extension).

In case of all models, the motion started from 20 degrees of flexion angle $(\alpha)$ (standing position) up to 120 degree of flexion angle (squat position). This was the first part of the squat, with downward motion. The flexion motion was followed by the extension of the knee joint, from 120 degrees of flexion angle back to 20 degrees of flexion angle.

\section{Introduction of wear imbalance}

In this study, one common (TW = total wear per million cycle) and three new wear parameters are introduced to characterize TKR wear: the relative lateral and relative medial wear ( $\left.\mathrm{RW}_{\text {lateral }}, \mathrm{RW}_{\text {medial }}\right)$, and most importantly, the wear imbalance (WIB).

Wear imbalance demonstrates as percentage how much medial wear deviates compared to lateral wear. It also implies that if a TKR is exposed to uneven medial load (and wear) than a so-called hollowing mechanism can commence on the above-mentioned TKR plateau (Fig. 5). In the long term, hollowing leads to the point 


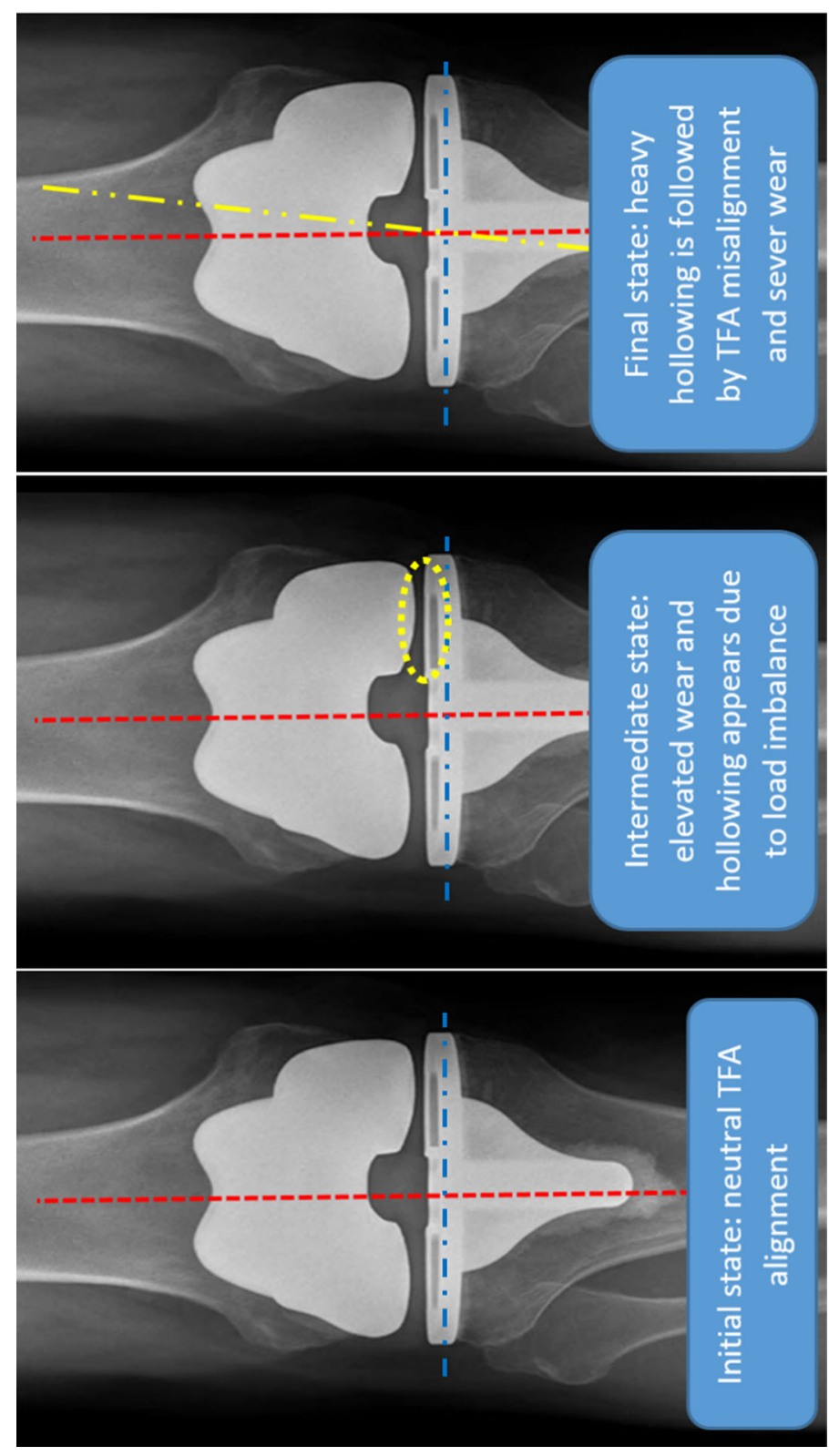

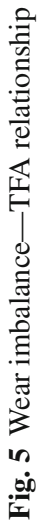


Table 3 Wear parameters of different TKRs

\begin{tabular}{lll}
\hline TKR & TW $\left[10^{-6} \mathrm{~mm}^{3} /\right.$ cycle] & Averaged WIB [\%] \\
\hline BioTech PS & $\sim 14.4$ & $\sim 2.3$ \\
BioTech CR & $\sim 18.7$ & $\sim 15.6$ \\
Prototype & $\sim 16.7$ & $\sim 5.9$ \\
\hline
\end{tabular}

that the physiological tibiofemoral alignment of the TKR will be tilted and this abnormal tilt becomes a wear-inducing factor [12].

In terms of the wear parameters, TW is defined as a number (at one million cycles), while $\mathrm{RW}_{\text {lateral }}$, $\mathrm{RW}_{\text {medial }}$ and WIB are determined a function of flexion angle $(\alpha)$ :

$$
\begin{aligned}
\mathrm{TW} & =\left(W_{\text {medial }}+W_{\text {lateral }}\right) \cdot 10^{6}, \\
\mathrm{RW}_{\text {lateral }}(\alpha) & =\frac{\mathrm{Wear}_{\text {lateral }}(\alpha)}{\operatorname{Wear}_{\text {total }}(\alpha)} \cdot 100, \\
\mathrm{RW}_{\text {medial }}(\alpha) & =\frac{\operatorname{Wear}_{\text {medial }}(\alpha)}{\mathrm{Wear}_{\text {total }}(\alpha)} \cdot 100, \\
\mathrm{WIB}(\alpha) & =\mathrm{RW}_{\text {medial }}(\alpha)-\mathrm{RW}_{\text {lateral }}(\alpha) .
\end{aligned}
$$

\section{Results}

The lateral and medial wear results, obtained from Eqs. (4) and (5), were incorporated into Eqs. (6, 7, 8, 9). Consequently, WIB functions for all TKRs were determined and plotted in Fig. 6. It is important to note that all of the obtained wear results are shown for one complete cycle (one squat movement: downwards and upwards).

In order to better qualify the eventual differences between the investigated TKRs, a quantitative comparison has been made with regard to total wear (medial wear + lateral wear) propagation (Fig. 7).

In addition, the averaged WIBs for full flexion-extension range, together with $T W$, have also been calculated and summarized in Table 3.

After having obtained these general results, all TKRs were examined separately.

First, PS TKR was analyzed concerning its wear propagation (Fig. 8). It was apparent from the results that this TKR provided the lowest total wear and the most balanced wear propagation among the other TKRs. Only $2.3 \%$ wear imbalance was present between the lateral and medial sides. It is notable that wear is generally higher at the medial contact plateau [34]; however, this is not a desired phenomenon.

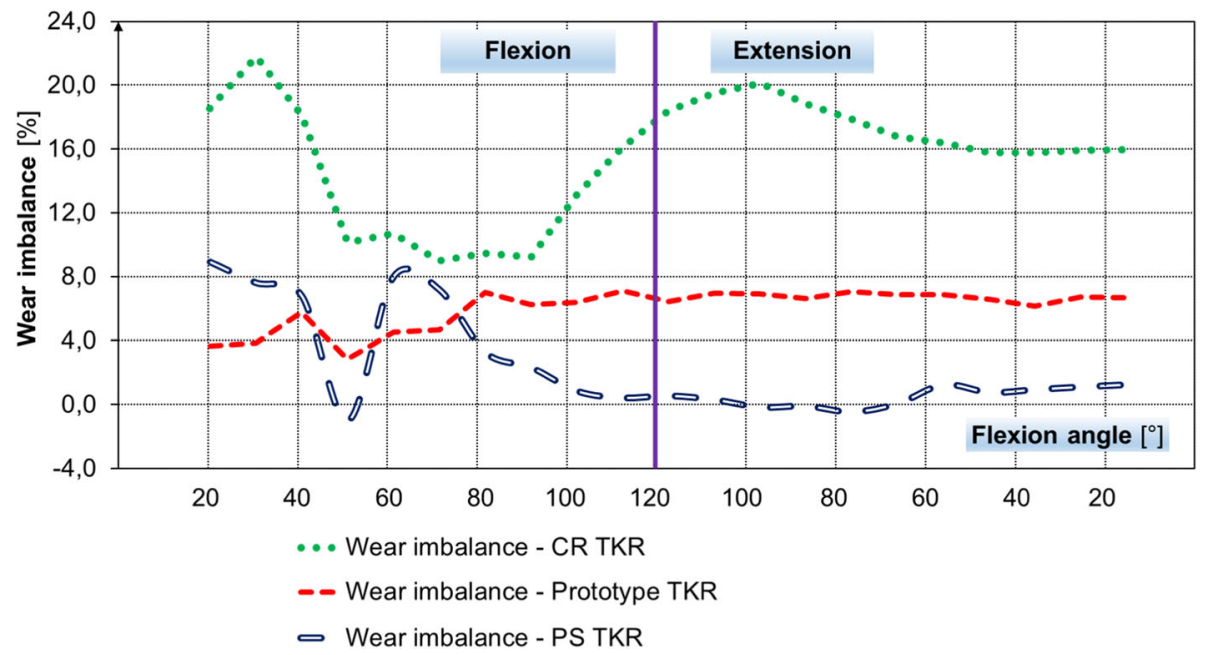

Fig. 6 Wear imbalance of different TKRs 


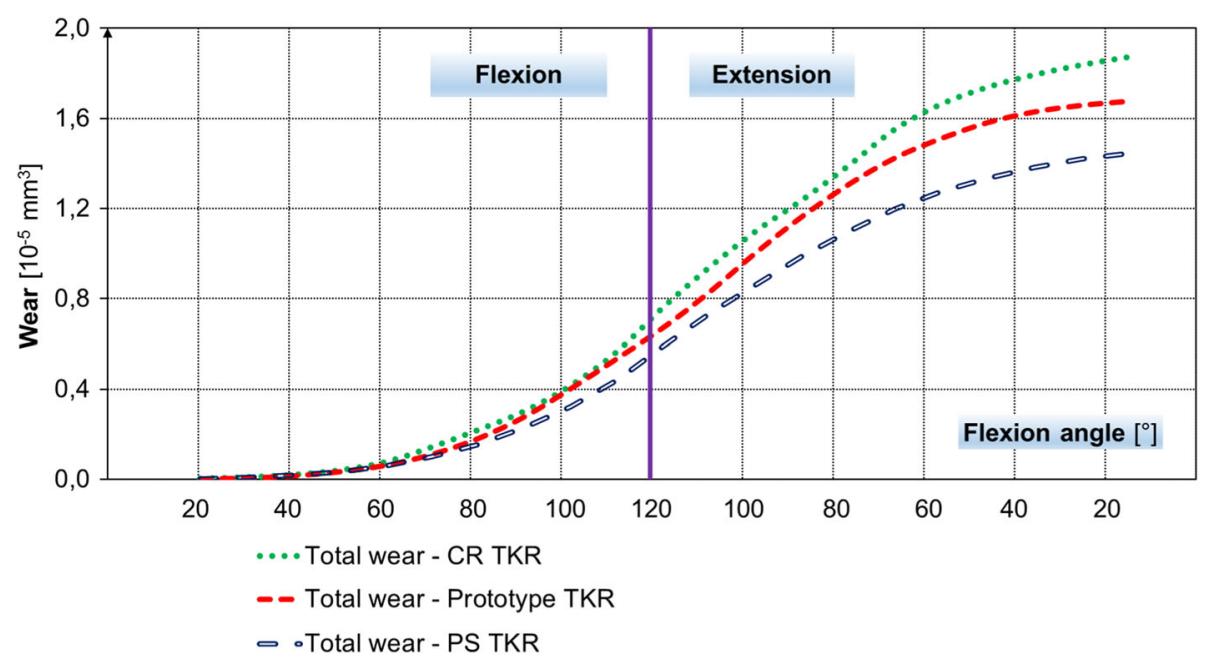

Fig. 7 Comparison of TKR wear propagation

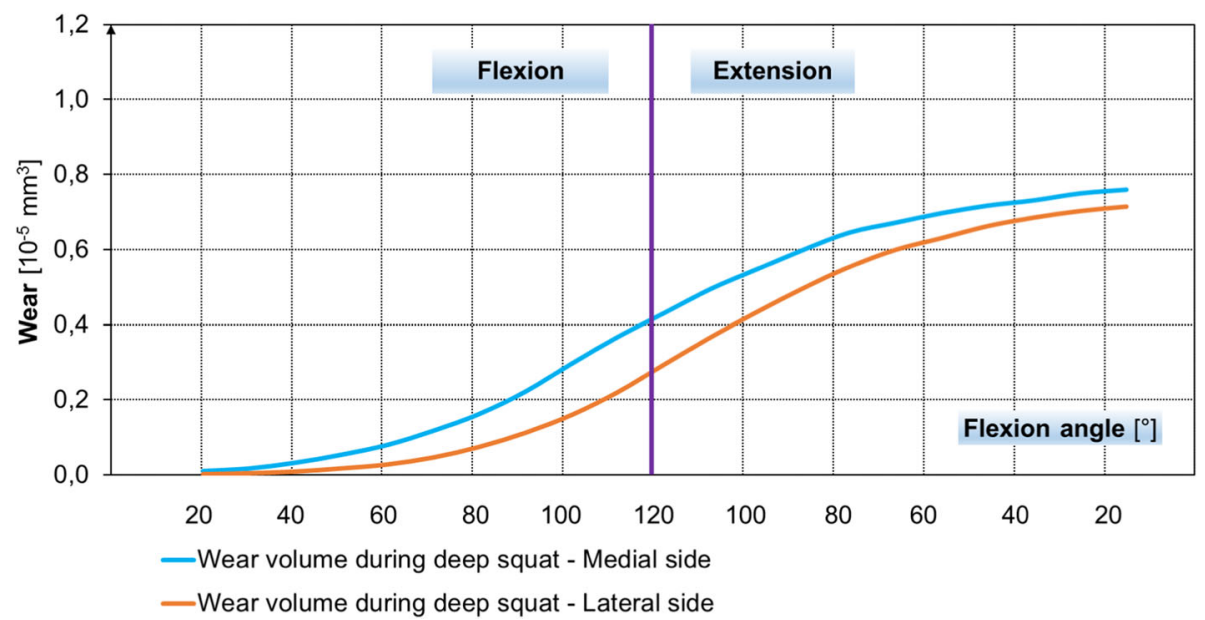

Fig. 8 Wear propagation of the Posterior Stabilized TKR

CR TKR was the next in the line. Here, obviously less balance could be observed between the two sides (Fig. 9). It must be mentioned that if the CR TKR is compared to the PS TKR, then over 20\% more total wear is present at CR, which make the PS TKR more superior with regard to its wear resistance ability.

Finally, wear of the prototype TKR was calculated (Fig. 10), where the lateral and medial wear were distributed more equally.

\section{Discussion}

With respect to wear, the aim "the lower the better" should generally be achieved; however, a balanced wear propagation on the lateral and medial sides of the tibial tray is also critically important. Feng et al. [35] quantitatively proved that an unbalanced medio-lateral alignment can be a direct cause of severe wear, leading to TKR failure in the periphery of the tibial tray, most likely at the medial side. By looking at the obtained results from the multibody dynamic simulations (Figs. 8, 9, 10), the tendency of higher wear propagation at the medial side can be observed.

Based on the numerically obtained total wear results, when compared to the total wear of other knee replacements from the literature [36], PS, CR and Prototype TKRs are situated in the middle range (Table 4). This comparison serves as a basic validation for the multibody dynamic simulation. 


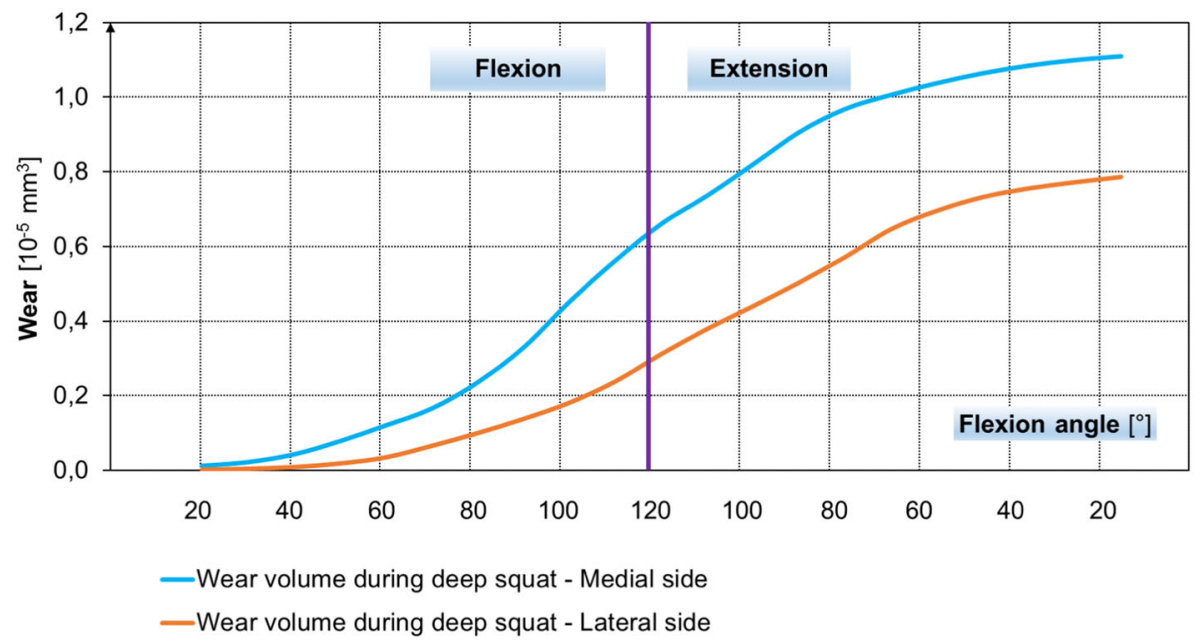

Fig. 9 Wear propagation of the Cruciate Retained TKR

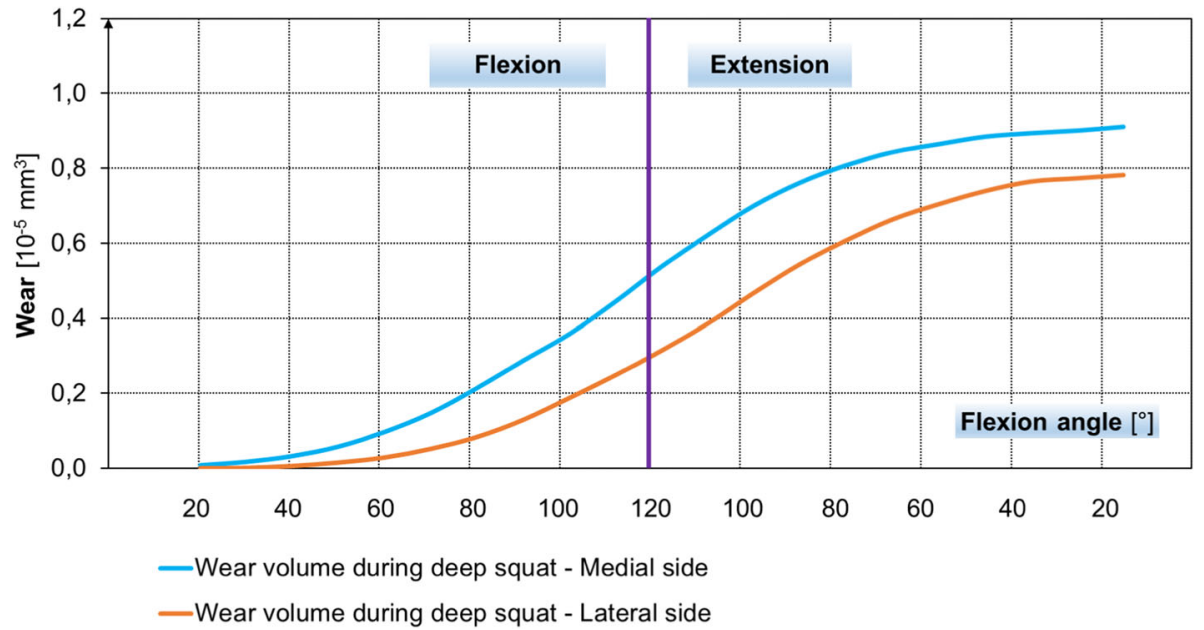

Fig. 10 Wear propagation of the Prototype TKR

Table 4 Comparison of $T W$ with regard to different TKRs

\begin{tabular}{ll}
\hline TKR & TW $\left[\mathrm{mm}^{3} /\right.$ million cycle $]$ \\
\hline BioTech PS & $\sim 14.4$ \\
BioTech CR & $\sim 18.7$ \\
Prototype & $\sim 16.7$ \\
Nexgen [36] & $\sim 14.9$ \\
PFC Sigma [36] & $\sim 17.8$ \\
Scorpio [36] & $\sim 6.6$
\end{tabular}

The wear results clearly pointed out that the Biotech PS TKR (Fig. 8) provided the lowest total wear with the least wear imbalance (approximately $2.3 \%$ averaged WIM). Such wear propagation on both sides of the tray can ensure that the "worn-through" stage would be postponed and implant revision could be significantly prolonged.

As we look at the following result (Fig. 9), the CR-type prosthesis experienced $\sim 23 \%$ higher total wear compared to the PS type. Even less favorable, the averaged lateral and medial wear imbalance was $\sim 15.6 \%$, which can lead to severe abrasion on the medial side and possible TKR retrieval before time.

A prototype TKR (Fig. 10), designed by the late Professor Gábor Krakovits [27], has also been included in the investigations, which brought forth the following results: this specific TKR produced only $13.7 \%$ more 
wear compared to the reference PS TKR, while the averaged wear imbalance between the lateral and medial side was only $5.9 \%$.

To have an adequately balanced wear on both sides, it would be required that the percentage difference should not exceed 5\%, which is a generally accepted level in engineering. As can be seen, only the PS could stay within such limits, while the Prototype TKR was close to it. Therefore, the CR-type TKR should be modified in its geometry in order to avoid generating high wear imbalance on its medial side.

From Fig. 6, it can be concluded that the Prototype and PS TKRs exhibit a low level of imbalance with small deviation throughout the full flexion-extension range of the knee joint (20 to 120 degrees of flexion angle). Based on these trends, it can be envisaged that athletic motions like walking $\left(0\right.$ to $\left.\sim 50^{\circ}\right)$, jogging $(0$ to $\sim 100^{\circ}$ ) or squatting $\left(\leqslant 120^{\circ}\right)$ will not fall into a segment where significantly elevated wear imbalance is found. Accordingly, these TKRs will not potentially be subjected to elevated wear due to tibiofemoral misalignment at the medial side.

As for the CR TKR, the results are more hectic compared to the Prototype or the PS TKR. The level of wear imbalance remains also considerably high during knee extension. This means that athletic motions, which are required to be carried out in the full flexion-extension range, can presumably lead to accelerated hollowing and wear in case of this specific TKR.

As a summary of the results, by the combination of multibody dynamic models with an augmented Archard's law, wear imbalance was introduced. This parameter can provide practical information about possible TKR failure due to uneven load distribution, which leads to hollowing and tibiofemoral misalignment, mainly at the medial contact plateaus. It should be emphasized that TFA determines load distribution between the contact plateaus; therefore, it is recommended to keep it neutral $\left(90^{\circ}\right)$ by having an optimal TKR geometry, otherwise it can increase wear even threefold [37].

Nonetheless, if the proposed method is taken into account by optimizing TKR geometry to closely constant numerical wear balance, then tibiofemoral misalignment can be significantly postponed or avoided in long term.

The limitation of the study must be also mentioned. Only three medium-sized TKRs were included into the numerical investigations; therefore, the obtained results can represent general trends about the lateral and medial wear propagation and imbalance, but they cannot provide correlation about the TKR size effect on wear. TKR size is known to be an important factor, since lately it has been statistically proven [38] that medium-sized TKRs can better restore specific knee-related kinematic parameters, e.g., the rotation than small-sized TKRs. In addition, the thickness of the tibial inserts has also been proven to be a contradictory parameter in knee biomechanics. Affatato et al. [39] demonstrated that higher wear ratios were found with large-sized tibial UHMWPE inserts than small- or middle-sized ones under the same kinetic and kinematic condition.

It is noteworthy to mention that this is conflicting theme since large implants have also large contact surfaces, which should provide lower contact pressure. As it is widely known, the precondition of low wear is low pressure.

Consequently, these unexpected and controversial results from the literature highlight the considerable effect of size on TKR wear and therefore on TKR functionality.

Due to the lack of studies regarding the influence of knee implant size (small, medium, large) on wear, as a next step, the above-mentioned numerical tool will be used to carry out several numerical simulations (min. $5 \mathrm{TKR} / \mathrm{size}$ ) to establish correlation on how TKR size influences wear propagation.

\section{Conclusions}

As a conclusion, a new hybrid model (combination of MBD and ODE) has been presented which allows us to investigate wear propagation on both sides of the tibia plateau. This model includes new parameters (time-dependent, non-constant slide-roll and contact forces) which have not yet been investigated or applied. As a result of this paper, the following possible implication is introduced: by the use of wear imbalance, it can be predicted whether a TKR is potentially subjected to failure due to subsequent hollowing followed by tibiofemoral misalignment. It should be recalled that tibiofemoral alignment is a major parameter that affects wear since it is responsible for distributing the load between the two condyles.

Acknowledgements This study was funded by the by Key Project of the National Social Science Foundation of China (19ZDA352), National Natural Science Foundation of China (No. 81772423), NSFC-RSE Joint Project (81911530253), National Key R\&D Program of China (2018YFF0300903, 2018YFF0300905), K. C. Wong Magna Fund in Ningbo University and the Eötvös Loránd University. 
Funding Open access funding provided by Eötvös Loránd University.

Open Access This article is licensed under a Creative Commons Attribution 4.0 International License, which permits use, sharing, adaptation, distribution and reproduction in any medium or format, as long as you give appropriate credit to the original author(s) and the source, provide a link to the Creative Commons licence, and indicate if changes were made. The images or other third party material in this article are included in the article's Creative Commons licence, unless indicated otherwise in a credit line to the material. If material is not included in the article's Creative Commons licence and your intended use is not permitted by statutory regulation or exceeds the permitted use, you will need to obtain permission directly from the copyright holder. To view a copy of this licence, visit http://creativecommons.org/licenses/by/4.0/.

\section{References}

1. Sharkey, P.F., Lichstein, P.M., Shen, C., Tokarski, A.T., Parvizi, J.: Why are total knee arthroplasties failing today-has anything changed after 10 years? J. Arthroplasty 29, 1774-1778 (2014)

2. McGloughlin, T., Kavanagh, A.: The influence of slip ratios in contemporary TKR on the wear of ultra-high molecular weight polyethylene (UHMWPE): An experimental view. J. Biomech. 31, 8 (1998)

3. Patten, E.W., Van Citters, D., Ries, M.D., Pruitt, L.A.: Quantifying cross-shear under translation, rolling, and rotation, and its effect on UHMWPE wear. Wear 313, 125-134 (2014)

4. Lopez-Cervantes, A., Dominguez-Lopez, I., Barceinas-Sanchez, J.D.O., Garcia-Garcia, A.L.: Effects of surface texturing on the performance of biocompatible UHMWPE as a bearing material during in vitro lubricated sliding/rolling motion. J. Mech. Behav. Biomed. Mater. 20, 45-53 (2013)

5. Rawal, B.R., Yadav, A., Pare, V.: Life estimation of knee joint prosthesis by combined effect of fatigue and wear. Procedia Technol. 23, 60-67 (2016)

6. Nägerl, H., Frosch, K.H., Wachowski, M.M., Dumont, C., Abicht, Ch., Adam, P., Kubein-Meesenburg, D.: A novel total knee replacement by rolling articulating surfaces. In vivo functional measurements and tests. Acta Bioeng. Biomech. 10, 55-60 (2008)

7. Fekete, G., De Baets, P., Wahab, M.A., Csizmadia, B.M., Katona, G., Vanegas-Useche, L.V., Solanilla, J.A.: Sliding-rolling ratio during deep squat with regard to different knee prostheses. Acta Polytechica Hungarica 9, 5-24 (2012)

8. Laurent, M.P., Johnson, T.S., Yao, J.Q., Blanchard, C.R., Crowninshield, R.D.: In vitro lateral versus medial wear of knee prosthesis. Wear 255, 1101-1106 (2003)

9. Massin, P.: How does total knee replacement technique influence polyethylene wear? Orthopaed. Traumatol. Surg. Res. 103, 21-27 (2017)

10. Innocenti, B., Fekete, G., Pianigiani, S.: Biomechanical analysis of augments in revision total knee arthroplasty. J. Biomed. Eng. 140, $111006(2018)$

11. Collier, M.B., Engh, C.A., Hatten, K.M., Ginn, S.T., Sheils, T.M., Engh, G.: Radiographic assessment of the thickness lost from polyethylene tibial inserts that had been sterilized differently. J. Bone Joint Surg. Am. 90, 1543-52 (2008)

12. Wasielewski, R.C., Galante, J.O., Leighty, R.M., Natarajan, R.N., Rosenberg, A.G.: Wear patterns on retrieved polyethylene tibial inserts and their relationship to technical considerations during total knee arthroplasty. Clin. Orthopaed. Related Res. 299, 31-43 (1994)

13. Fekete, G., Sun, D., Gu, Y., Neis, P.D., Ferreira, N.F., Innocenti, B., Csizmadia, B.M.: Tibiofemoral wear in standard and non-standard squat: implication for total knee arthroplasty. Muscle Ligaments Joints J. 7, 520-528 (2017)

14. Fekete, G., Bíró, I., Csizmadia, B.M.: Mechanical model for estimating wear in tibio-femoral connection in total knee replacements. Biomechanica Hungarica 10(1), 55-63 (2017)

15. Mell, S.P., Wimmer, M.A., Lundberg, H.J.: The choice of the femoral center of rotation affects material loss in totalknee replacement wear testing - a parametric finite element study ofISO 14243-3. J. Biomech. 88, 104-112 (2019)

16. Archard, J.F., Hirst, W.: The wear of metals under unlubricated conditions. Proc. R. Soc. A 236, 397-410 (1956)

17. Pal, S., Haider, H., Laz, P.J., Knight, L.A., Rullkoetter, P.J.: Probabilistic computational modeling of total knee replacement wear. Wear 264(7-8), 701-707 (2008)

18. O’Brien, S.T., Bohm, E.R., Petrak, M.J., Wyss, U.P., Brandt, J.-M.: An energy dissipation and cross shear time dependent computational wear model for the analysis of polyethylene wear in total knee replacements. J. Biomech. 47, 1127-1133 (2014)

19. Turell, M., Wang, A., Bellare, A.: Quantification of the effect of cross-path motion on the wear rate of ultra-high molecular weight polyethylene. Wear 255, 1034-1039 (2003)

20. Abdelgaied, A., Liu, F., Brockett, C., Jennings, L., Fisher, J., Jin, Z.: Computational wear prediction of artificial knee joints based on a new wear law and formulation. J. Biomech. 44, 1108-1116 (2011)

21. Flores, P.: Modeling and simulation of wear in revolute clearance joints in multibody systems. Mech. Mach. Theory 44, 1211-1222 (2009)

22. Fekete, G., Csizmadia, B.M., Wahab, M.A., De Baets, P., Vanegas-Useche, L.V., Bíró, I.: Patellofemoral model of the knee joint under non-standard squatting. Dyna Colombia 81, 60-67 (2014)

23. Liu, G., Fekete, G., Yang, H., Ma, J., Sun, D., Mei, Q., Gu, Y.: Comparative 3-dimensional kinematic analysis of snatch technique between top-elite and sub-elite male weightlifters in 69-kg category. Heliyon 4, e00658 (2018)

24. Slater, L.V., Hart, J.M.: Muscle activation patterns during different squat techniques. J. Strength Condition. Res. 31, 667-676 (2017)

25. Slater, L.V., Hart, J.M.: The influence of knee alignment on lower extremity kinetics during squats. J. Electromyography Kinesiol. 31, 96-103 (2016)

26. Montes-Seguedo, J.L., Garcia-Garcia, A.L., Barceinas-Sanchez, J.D.O., Sosa-Savedra, J.C., Morales-Garcia, M.R.J., Gonzalez-Jasso, E., Dominguez-Lopez, I.: Mapping the friction coefficient of AISI 316L on UHMWPE lubricated with 
bovine serum to study the effect of loading and entrainment at high values of sliding-to-rolling ratio. Health Technol. 10, 385-390 (2020)

27. Krakovits, G.: Knee surface replacement prosthesis. U.S. Patent 6,344,059 B1, issued February 5 (2002)

28. Ling, Z.-K., Guo, H.-Q., Boersma, S.: Analytical study on the kinematic and dynamic behaviors of the knee joint. Med. Eng. Phys. 19, 29-36 (1997)

29. Momersteeg, T.J.A., Blankevoort, L., Huiskes, R., Kooloos, J.G.M., Kauer, J.M.G., Hendriks, J.C.M.: The effect of variable relative insertion orientation of human knee bone-ligament-bone complexes on the tensile stiffness. J. Biomech. 28, 745-752 (1995)

30. Walpole, S.C., Prieto-Merino, D., Edwards, P., Cleland, J., Stevens, G., Roberts, I.: The weight of nations: an estimation of adult human biomass. BMC Public Health 12(439), 1-6 (2012)

31. http://www.ksh.hu/docs/hun/xftp/stattukor/egeszsegallapot1617.pdf. Accessed 16 Jan 2020

32. Mason, J.J., Leszko, F., Johnson, T., Komistek, R.D.: Patellofemoral joint forces. J. Biomech. 41, 2337-2348 (2008)

33. Innocenti, B., Pianigiani, S., Labey, L., Victor, J., Bellemans, J.: Contact forces in several TKA designs during squatting: a numerical sensitivity analysis. J. Biomech. 44, 1573-1581 (2011)

34. Tone, S., Hasegawa, M., Naito, Y., Pezzotti, G., Sudo, A.: Raman spectroscopy reveals creep and wear rate of e-beam-sterilized conventional UHMWPE tibial inserts. J. Mech. Behav. Biomed. Mater. 110, 109302 (2020)

35. Feng, E.L., Stulberg, S.D., Wixson, R.L.: Progressive subluxation and polyethylene wear in total knee replacements with flat articular surfaces. Clin. Orthopaed. Related Res. 299, 60-71 (1994)

36. Okazaki, Y., Hosoba, M., Miura, S., Mochizuki, T.: Effects of knee simulator control method and radiation dose on UHMWPE wear rate, and relationship between wear rate and clinical revision rate in National Joint Registry. J. Mech. Behav. Biomed. Mater. 90, 182-190 (2019)

37. Gromov, K., Korchi, M., Thomsen, M.G., Husted, H., Troelsen, A.: What is the optimal alignment of the tibial and femoral components in knee arthroplasty? Acta Orthopaedica 85, 480-487 (2014)

38. Fekete, G., Sun, D., Liu, G., Gu, Y., Balassa, G.P., Bíró, I., Neis, P.D., Jánosi, E.: Preliminary results of size and slide-roll effect on the kinematics of Total Knee Replacements. Acta Polytechnica Hungarica 15, 143-153 (2018)

39. Affatato, S., Grillini, L., Battaglia, S., Taddei, P., Modena, E., Sudanese, A.: Does knee implant size affect wear variability? Tribol. Int. 66, 174-181 (2013)

Publisher's Note Springer Nature remains neutral with regard to jurisdictional claims in published maps and institutional affiliations. 